\title{
O protagonismo de idosas na promoção da saúde: rodas de conversa na comunidade
}

\section{The role of older women in health promotion: Conversation wheels in the community}

\author{
Camila Fabiana Lemos Francescato', Vivian Steffen Heimerdinger², Valéria Baccarin laniski³, \\ Arlete Regina Roman ${ }^{4}$
}

' Enfermeira, residente do Programa de Residência Multiprofissional em Saúde da Família UNIJUÍ/FUMSSAR. Universidade Regional do Noroeste do Estado do Rio Grande do Sul-UNIJUí. <camilaf.lemos@bol.com.br>

${ }^{2}$ Psicóloga, preceptora de campo do Programa de Residência Multiprofissional em Saúde da Família UNIJUÍ/FUMSSAR. Fundação Municipal de Saúde de Santa Rosa _FUMSSAR.<fabio.turra@yahoo.com.br>

${ }^{3}$ Nutricionista, residente do Programa de Residência Multiprofissional em Saúde da Família UNIJUÍ/FUMSSAR. Universidade Regional do Noroeste do Estado do Rio Grande do Sul - UNIJUÍ. <valeriaianiski@yahoo.com.br>

${ }^{4}$ Enfermeira, orientadora tutora do Programa de Residência Multiprofissional em Saúde da Família UNIJUÍ/FUMSSAR. Universidade Regional do Noroeste do Estado do Rio Grande do Sul-UNIJUÍ. <arleter@unijui.edu.br>

\section{ARTICLE INFO}

\section{Article history}

Received: 23/07/2017

Accepted: 11/10/2017

\section{Correspondent Author}

Valéria Baccarin laniski

Rua Clódio Beck, 516 - Centro

98855-000 Entre-ljuís, RS, Brasi

<valeriaianiski@yahoo.com.br>

\section{(C) 2017 All rights reserved}

\section{Editors}

Alfredo Cataldo Neto

Paula Engroff

\begin{abstract}
RESUMO
O objetivo deste estudo foi compreender como usuárias que frequentam rodas de conversa na comunidade exercem o protagonismo, utilizando o conhecimento adquirido nas rodas e de que maneira multiplicam essas informações na promoção do cuidado em saúde. Trata-se de uma pesquisa qualitativa, descritiva que entrevistou oito mulheres idosas (60 anos ou mais) da comunidade, pertencentes a diferentes micro áreas de um território de saúde, de um município do interior do estado do Rio Grande do Sul/BR, durante o mês de setembro de 2016. As mesmas deveriam ter participado de no mínimo quatro rodas de conversa no decorrente ano. Elas responderam a um questionário com perguntas abertas e fechadas. As entrevistas foram analisadas segundo os pressupostos da análise de conteúdo proposta por Minayo (2010) e os resultados foram organizados por categorias de análise, considerando as etapas de pré-análise, exploração do material e tratamento e interpretação dos resultados. Conclui-se que as rodas de conversa enquanto metodologia ativa e tecnologia leve do cuidado geram benefícios na promoção do autocuidado, transformando o cotidiano dos indivíduos que delas participam e o meio social e familiar ao qual estão inseridas, ocupando elas, o papel de agentes multiplicadores de saberes nos seus espaços de convívio. Além disso, o espaço da roda de conversa mostrou-se um local de construção coletiva, que eleva relações harmoniosas com a equipe de saúde, fortalecendo vínculos terapêuticos e o protagonismo das usuárias envolvidas.
\end{abstract}

PALAVRAS-CHAVE: atenção primária à saúde; idoso; autocuidado; estrutura de grupo; autonomia pessoal.

\begin{abstract}
The purpose of this study was to understand how users who frequent talk in the community exercised the leading role, using the knowledge acquired in the wheels and how they multiply this information in the promotion of health care. This is a qualitative, descriptive research that interviewed eight elderly women ( 60 years old or more) of the community, belonging to different micro areas of a health territory, from a municipality in the interior of the state of Rio Grande do Sul/BR, during The month of September 2016. They should have participated in at least four rounds of conversation in the coming year. They answered a questionnaire with open and closed questions. The interviews were analyzed according to the assumptions of the content analysis proposed by Minayo (2010) and the results were organized by categories of analysis, considering the steps of pre-analysis, material exploration and treatment and interpretation of the results. It is concluded that the wheels of conversation as active methodology and light technology of care generate benefits in the promotion of self-care, transforming the daily life of the individuals who participate and the social and family environment to which they are inserted, occupying them, the role of multiplier agents Of knowledge in their venues. In addition, the space of the conversation wheel was a collective construction site, which elevates harmonious relations with the health team, strengthening therapeutic ties and the protagonism of the users involved. KEYWORDS: primary health care; elderly; self-care; group structure; personal autonomy.
\end{abstract}




\section{INTRODUÇÃO}

A Atenção Primária em Saúde (APS) é reconhecidamente um componente-chave do sistema de saúde brasileiro. Esse reconhecimento fundamenta-se nas evidências de seu impacto na saúde e no desenvolvimento da população, tendo como resultado melhores indicadores de saúde, maior eficiência no fluxo dos usuários dentro do sistema, tratamento mais efetivo de condições crônicas, maior eficiência do cuidado, maior utilização de práticas preventivas, maior satisfação dos usuários e diminuição das injustiças sobre o acesso aos serviços e o estado geral de saúde. ${ }^{1}$

A proposta de mudança no modelo de saúde sugeriu a criação de uma rede de assistência à saúde que abrangesse a população, sendo instituídas algumas diretrizes, respeitando as realidades regionais, municipais e locais, além da criação das Unidades Básicas de Saúde.

No Brasil a atual situação de saúde, caracterizada pela transição demográfica acelerada e por uma situação epidemiológica de tripla carga de doenças, aumenta progressivamente a incidência de hipertensão arterial e os transtornos depressivos. ${ }^{2}$ Uma população em processo rápido de envelhecimento constitui um crescente aumento das doenças crônicas, visto que estas afetam mais os segmentos de maior idade. ${ }^{3,4}$

Um dos desafios do Sistema Único de Saúde (SUS) é desenvolver um cuidado voltado para a promoção da saúde, desenvolvendo atividades que visem mudanças no estilo de vida, através da prática da atividade física bem como uma alimentação saudável. ${ }^{4}$ Para isso, precisa-se despertar para o uso das tecnologias leves de cuidado. O uso das tecnologias leves do cuidado ocorre através da escuta ampliada, do acolhimento e da formação do vínculo. Merhy ${ }^{5}$ nos explica que estes aspectos fortalecem o protagonismo dos sujeitos envolvidos na construção do seu cuidado, o que se torna essencial no processo de trabalho multidisciplinar, promovendo a corresponsabilidade e a cogestão do cuidado.

O trabalho em saúde possui particularidades, sendo construído dinamicamente por meio das relações cotidianas entre os usuários e os profissionais de saúde. A educação em saúde, neste sentido é um dos pilares para a promoção e prevenção de agravos à saúde, que é também o que preconiza a Política Nacional de Atenção Básica. ${ }^{6}$

A Educação em Saúde constitui parte essencial na promoção da saúde e na prevenção de doenças, como também contribui para o tratamento precoce e eficaz das enfermidades, minimizando o sofrimento e a incapacidade, atuando sobre o conhecimento das pessoas e a capacidade de intervir sobre suas vidas. ${ }^{?}$

O Método da Roda, também conhecido como Método Paidéia proposto por Campos, ${ }^{8}$ é um espaço de politização da gestão, porque rompe com o modelo burocrático de gestão, uma vez que se sintoniza com muitas tradições libertárias da educação e da política, ideia permanente de coprodução e construção de autonomia dos sujeitos, visando à democratização das relações de poder, constituindo, ao mesmo tempo, espaços de ensino-aprendizagem, de elaboração e de organização de processos de trabalho e de atenção às subjetividades, desejos e relações interpessoais.

A aplicação dessa metodologia, no território, é proporcionada pelo trabalho desenvolvido no Programa de Residência Multiprofissional em Saúde da Família (PRMSF). O PRMSF constitui uma modalidade de ensino de pós-graduação Lato Sensu, em regime de dedicação exclusiva, com duração de dois anos. As residências multiprofissionais e em área profissional da saúde, foram criadas a partir da promulgação da Lei n⿳⺈ 11.129 de $2005,{ }^{9}$ são orientadas pelos princípios e diretrizes do SUS, a partir das necessidades e realidades loco regionais, e abrangem as profissões da área da saúde, a saber: Biomedicina, Ciências Biológicas, Educação Física, Enfermagem, Farmácia, Fisioterapia, Fonoaudiologia, Medicina Veterinária, Nutrição, Odontologia, Psicologia, Serviço Social e Terapia Ocupacional.

Esta modalidade de ensino possibilita e valoriza a vivência da interdisciplinaridade, com a perspectiva inerente ao cuidado em saúde e aos princípios do SUS. $\mathrm{O}$ agir em conjunto torna mais eficaz a resolução dos problemas recorrentes nas realidades enfrentadas pelos profissionais de saúde. Trabalhar em equipe proporciona aprendizado mútuo e uma assistência de qualidade, à medida que enxerga o indivíduo sob diferentes perspectivas, tornando o ensino, a aprendizagem e a troca de saberes um processo dinâmico e leve, que envolve a integração de diversos saberes. ${ }^{10}$

A proposta das rodas foi apresentada pelos preceptores de campo a equipe de saúde e ao programa de residência, para ser realizada nos territórios com cobertura de Agente Comunitário de Saúde (ACS). As rodas foram implementadas no ano de 2010, com encontros mensais em cada micro área, de um território de saúde, que abrange uma população de 7.160 pessoas de acordo com levantamento interno do município, têm cobertura de ACS de 30\% e aproximadamente 302 pessoas hipertensas e 401 diabéticas.

Inserida esta metodologia de trabalho na comunidade, como exemplo de uma tecnologia leve no cuidado, pode-se difundir um tensionamento 
para a mudança no modelo de atenção utilizado (modelo biomédico), para um modelo mais holístico e participativo, onde o usuário é protagonista na construção do seu cuidado.

Partindo dessa premissa, o estudo teve como objetivo compreender a partir da percepção de usuárias sobre as rodas de conversa, como elas exercem seu protagonismo, utilizando o conhecimento adquirido através desses encontros e de que maneira multiplicam essas informações na promoção do cuidado.

\section{MÉTODO}

Este estudo trata-se de uma pesquisa qualitativa do tipo descritiva. A amostra foi constituída por mulheres, usuárias de uma Unidade Básica Saúde da Família, localizada em um município do noroeste do estado do Rio Grande do Sul e que pertenciam a alguma das quatro microáreas desse território. Para manter o anonimato das entrevistadas, as mesmas foram identificadas nesta pesquisa com nome de flores, Margarida, Violeta, Rosa, Orquídea, Bromélia, Astromélia, Tulipa e Cravo.

A coleta de dados ocorreu por meio de questionário semiestruturado, com perguntas abertas e fechadas, aplicado após as Rodas de Conversa no período de setembro de 2016. A análise das entrevistas ocorreu com base nos pressupostos da técnica de análise de conteúdo proposta por Minayo ${ }^{11}$ e os resultados foram organizados por categorias de análise, considerando as etapas de pré-análise, exploração do material e tratamento e interpretação dos resultados.

Foram incluídas mulheres com idade igual ou superior a 60 anos, participação no mínimo de quatro encontros anuais nas Rodas de Conversa e que aceitaram participar da pesquisa, confirmado por meio da assinatura do Termo de Consentimento Livre e Esclarecido.

As rodas de conversa eram encontros mensais que ocorriam entre profissionais de saúde e a comunidade, em cada microárea de um território de saúde, com duração de aproximadamente uma hora e trinta minutos. $\mathrm{O}$ encontro se baseava na escuta, acolhimento e formação do vínculo, fortalecendo o protagonismo dos sujeitos envolvidos na construção do cuidado, o que é essencial no processo de trabalho multidisciplinar e promove a corresponsabilidade e a cogestão do cuidado. As rodas, surgiram da necessidade de mudança no modelo de atenção à saúde ofertado a população - saída de um modelo biomédico, centrado na cura das doenças para um modelo holístico, baseado na integralidade do sujeito e na longitudinalidade do cuidado - sendo caracterizada, como uma tecnologia leve do cuidado. ${ }^{5}$
Esta pesquisa seguiu todos os preceitos éticos de uma pesquisa com seres humanos preconizados pela Resolução no 466/2012 do Conselho Nacional da Saúde/Ministério da Saúde e foi aprovada pelo Comitê de Ética em Pesquisa da UNIJUÍ com o CAAE $\mathrm{n}$ o 57714716.9.0000.5350 e parecer consubstanciado no 1.727 .816 .

\section{RESULTADOS E DISCUSSÃO}

A amostra deste estudo foi composta por oito usuárias, com idade entre 60 e 80 anos de idade e todas possuíam filhos. Quanto à situação conjugal verificou-se que quatro, são casadas, duas divorciadas e duas viúvas. Duas moram sozinhas, duas moram com os filhos e quatro moram com companheiro.

Em conversa informal as idosas que são viúvas e divorciadas, relataram que após ficarem sem companhia marital, preferiram dedicar-se aos filhos e netos, lhes assessorando nas atividades do ambiente familiar. A localização da residência na proximidade de membros da família, serviço e transporte podem significar a diferença entre uma interação social positiva e o isolamento.

A maioria das participantes apresenta ocupação relacionada com as atividades de cuidado do lar, destacando que já estão aposentadas, sendo apenas duas entrevistadas que desenvolvem atividades autônomas do tipo: manicure e cabeleireira. Devido à maior parte delas estarem aposentadas, permite que as mesmas direcionem maior tempo para o seu cuidado e participação em espaços sociais.

Quanto à escolaridade, observou-se que entre as entrevistadas três apresentam ensino fundamental incompleto, quatro ensino médio incompleto e uma ensino médio completo. Nas entrevistas quando questionadas sobre a escolaridade, as mesmas justificaram-se em ter passado a infância na zona rural onde o acesso à educação era difícil, sendo priorizado aos homens da família, ficando elas responsáveis pelo cuidado do lar e irmãos mais novos.

Os baixos níveis de instrução e o analfabetismo estão associados a maior vulnerabilidade e morte durante o processo de envelhecimento, gerando impacto significativo no cotidiano, pois traz implicações sociais como a diminuição da participação social, empoderamento e mobilização política. Fica evidente que muitos elementos do meio social são os grandes responsáveis por afetar a qualidade de vida da população, como as condições de trabalho, renda, moradia e educação. ${ }^{1}$

Tais determinantes sociais acabam influenciando o processo de autonomia, fazendo com que essas mulheres atuem na sociedade de forma discreta contribuindo para o menor empoderamento. Desta 
maneira, ações e intervenções que incentivem a reflexão, criticidade e o engajamento social, fortalecem uma postura de protagonismo.

Protagonista e protagonismo são palavras comumente utilizadas no campo da literatura, empregadas para se referir a personagens de uma história, responsáveis pelo desenrolar de um enredo. ${ }^{12}$ No campo das artes cênicas, mais especificamente nos romances da teledramaturgia, protagonista é o personagem principal de uma história, usualmente interpretado por artistas amplamente conhecidos. Já no campo das ciências sociais, estes termos têm sido utilizados como variáveis de "sujeito" para designar grupos ou conjuntos de atores sociais que desencadeiam ações e se colocam ativamente na construção da história. ${ }^{11}$

Ao abordar nesta pesquisa a participação social de idosas, bem como o papel social que elas desenvolvem na comunidade, elegeu-se duas categorias temáticas ' $O$ protagonismo como meio' e 'O protagonismo como fim'.

\section{O protagonismo como meio*}

Produzir cuidado por meio do trabalho em saúde depende da construção de processos relacionais entre gestores e trabalhadores, trabalhadores e usuários que possam lidar com a exposição das implicações que a produção de cuidado opera..$^{5}$ O Método da Roda evidencia como o uso dessa tecnologia pode modificar o cotidiano dos atores envolvidos. ${ }^{13}$

A partir das entrevistas realizadas com as idosas, todas foram unânimes em dizer que se interessam pelas temáticas abordadas, bem como afirmaram compreender os temas na forma como são expostos. As temáticas das rodas são sempre escolhidas pelas usuárias, e nem sempre se relacionam diretamente com a saúde, mas também com lazer, interação social, profissões e questões pessoais.

Quando questionadas se as discussões realizadas acrescentam algo em seu dia a dia, todas afirmaram que sim, e na percepção do entrevistador as mesmas mostram-se receptivas e abertas a novos saberes e práticas. Fragmentos das falas de Margarida, Violeta, Rosa e Cravo exemplificam tal satisfação, a valorização deste espaço de convivência, troca de saberes e reflexão sobre suas ações. "Assim, através das dúvidas e troca de conversa consigo repensar os assuntos." Margarida. "Sim, em tudo, tanto na convivência e troca de experiência." Violeta. "A gente sempre aprende alguma coisa nova... Acrescenta muito nas atividades que eu realizo em casa, no cuidado comigo." Rosa. "Hoje me cuido muito melhor." Cravo.

Segundo Paulo Freire, ${ }^{14} \mathrm{O}$ processo de conscientização ocorre à medida que o sujeito reflete, sendo

\footnotetext{
*É um recurso, uma possibilidade, que tende a ser possível.
}

esses espaços locais, lugares de troca de saberes e cenários importantes para essa construção coletiva.

[...] Enquanto amadurecimento do ser para si, é processo, é vir a ser. Não ocorre em data marcada. É neste sentido que uma pedagogia da autonomia tem de estar centrada em experiências estimuladoras da decisão e da responsabilidade, vale dizer, em experiências respeitosas da liberdade..$^{14,41}$

Quando autorreferido algum tipo de problema de saúde, a maioria das participantes citou hipertensão arterial e transtornos depressivos. A hipertensão arterial na pessoa idosa traz impactos significativos na sua qualidade de vida, sendo uma das patologias mais incapacitantes, aumenta a morbimortalidade devido a dificuldades encontradas na adesão ao tratamento e a mudança de estilo de vida. ${ }^{2,3}$

A mudança natural do corpo durante o envelhecimento torna a depressão um dos transtornos psiquiátricos mais comuns entre as pessoas idosas, tendo sua maior prevalência em mulheres idosas. Isso acaba ocasionando impactos negativos, podendo gerar comprometimento físico, social e funcional, afetando a qualidade de vida dos idosos. ${ }^{15}$

Muitas participantes relataram se sentirem depressivas devido às perdas familiares ocorridas ao longo da vida e a solidão, resultando em pouco convívio social e inserção em espaços da sociedade. Apesar disso, muitas delas também relataram que participam de grupos ofertados pela unidade de saúde como o de alongamento e atividade física e também encontros de novena. Uma vez que há um antagonismo entre a solidão e relato de tempo para sair e se distrair.

Segundo Lopes, ${ }^{16}$ a solidão provoca um sentimento de vazio interior e pode estar presente em todas as fases da vida, sendo mais frequente com o envelhecimento. O envelhecimento ativo depende de uma diversidade de fatores e determinantes que envolvem indivíduos e famílias. ${ }^{17}$

As escolhas realizadas no presente terão impacto no futuro, pois o envelhecimento é um processo fisiológico natural, todavia, as atitudes e o padrão de vida adotado durante toda a vida irá refletir na ocorrência de comorbidades ou não nesta fase. Neste contexto, ao encarar discussões e escutar orientações e relatos sobre temas de sua rotina essas usuárias desmistificam crenças errôneas, ensaiam condutas mais saudáveis e exercem seu protagonismo como meio.

\section{O protagonismo como fim**}

Protagonismo como fim é visto, como um modo de saber, de se empoderar e fazer, é através dele que

** Objetivo para o qual se tem a intenção de concluir. 
se consegue transformar a informação recebida e multiplicar no cotidiano, nos espaços vividos.

"Empowerment" ou Empoderamento como tem sido traduzido no Brasil, não tem caráter universal. Referese ao processo de mobilizações e práticas destinadas a promover e impulsionar grupos e comunidades no sentido de seu crescimento, autonomia, melhora gradual e progressiva de suas vidas. ${ }^{18}$ Quando perguntadas se as informações abordadas nas rodas de conversa são utilizadas para o seu autocuidado e de que maneira, todas as usuárias referem que sim, e os cuidados com hábitos alimentares saudáveis são os mais evidentes. "Sim, alimentação e cuidado com o corpo." Rosa. "Sim, como proceder diferente na alimentação entre outras." Bromélia. "Sim, em tudo, desde o uso do chá até a ingestão de água." Orquídea. "Sim, na autoestima, na alimentação em me cuidar melhor." Astromélia.

Conforme Meneguessi, ${ }^{19}$ a teoria do autocuidado proposta por Dorotéia Oren, importante teorista de enfermagem, focaliza no indivíduo e na família para manter a vida, a saúde e bem estar, estando relacionada com as atividades do cotidiano como a ingestão de água e alimentos. Os depoimentos mostram que as informações repassadas nas rodas de conversa proporcionam a promoção do autocuidado, que vai desde o cuidado alimentar através de uso de chás e ingestão de água até o cuidado com o corpo.

Segundo Sampaio et al..$^{20}$ é nesse espaço de interação social que se tenciona a construção de novas possibilidades para o pensar, num movimento contínuo de perceber, refletir, agir, modificar, onde os participantes podem se reconhecer como condutores de sua ação e da sua própria possibilidade de "ser mais". Endossando essas ideias, Paulo Freire ${ }^{14: 88}$ nos diz que: "Mudar é difícil, mas necessário e possível".

Trabalhar as ações de promoção em saúde com as usuárias idosas tem possibilitado mudanças em seu cotidiano, contribuindo na melhora da qualidade de vida. Todas as participantes deste estudo, responderam que as informações fornecidas ajudaram na melhora da sua saúde, de sua família e círculo de amizades.

Quando questionadas sobre as informações que são repassadas serem utilizadas como forma exclusiva de autocuidado, sem acompanhamento de profissional de saúde, seis usuárias responderam que buscam ajuda de profissionais de saúde para complementar seu cuidado, no entanto, Cravo e Tulipa, responderam que "[...] consulto quando preciso, mas primeiro tento me cuidar sozinha. Não gosto muito de ir ao médico." Cravo. "Uso a maior parte das informações pro meu cuidado. O médico assim, só vou quando preciso muito." Tulipa.

O acesso e a utilização dos serviços de saúde dependem de um conjunto de fatores que podem ser divididos em determinantes da oferta e determinantes da demanda, isso é o que interfere no modo e na quantidade de consumo de serviços de saúde, no âmbito populacional. ${ }^{21}$

Desde que o saber cuidar do outro foi expropriado da sociedade e configurado em determinadas técnicas produzidas por determinados atores legalmente reconhecidos como tais, exercidas em determinados lugares e acessível somente a alguns, a dinâmica da vida se reduziu a estar ou não estar doente e esta situação definida com base em normas que determinam o normal e o patológico. $\mathrm{O}$ agenciamento da saúde, como ausência de doença, e desta, como sendo a falta de algo para fazer a máquina humana funcionar (o corpo), reproduz, no imaginário popular, a ideia de saúde restrita ao consumo de serviços e de medicamentos. ${ }^{22: 402}$

O desenvolvimento de ações voltadas à promoção da saúde aliando o saber popular com o saber científico auxiliam na diminuição do consumo dos serviços de saúde. A educação popular em saúde compreende e reconhece a importância dos saberes e práticas populares e sugere a mediação entre os saberes técnicocientíficos e populares, promovendo visibilidade e autonomia aos sujeitos junto à sociedade e aos serviços de saúde. ${ }^{23}$

Os assuntos abordados nesse espaço de troca de saberes e diálogo são elencados pelas usuárias, isso faz com que algumas dúvidas sobre determinadas patologias e cuidados em saúde sejam sanadas, refletindo em uma menor procura aos serviços de saúde. Ao serem questionadas se as informações adquiridas são repassadas para outras pessoas de sua convivência e de que maneira, todas responderam de forma afirmativa, o que podemos identificar nas falas que seguem. "Comento com a minha família, amigos e vizinhas." Tulipa, Margarida, Violeta. "Sempre converso com minhas amigos, minha família e também no grupo de atividade física." Cravo. "Eu sempre comento as coisas que aprendo... até com as clientes que eu atendo no salão." Astromélia, Bromélia.

Por meio das falas, percebe-se que as usuárias utilizam o cuidado consigo e com familiares no seu dia a dia. Supõe- se que modificam a si, ao ambiente e as práticas familiares bem como, os espaços sociais que convivem.

Interagir socialmente, sobretudo com amigos da mesma geração possibilita ao idoso a construção de novos laços de relação e favorece o bem estar físico, mental e social. ${ }^{24}$ Essas usuárias, como agentes de transformação nos espaços onde circulam, conseguem a partir da educação popular multiplicar o conhecimento adquirido, e assim, exercer seu protagonismo como um fim. 
Patrocínio ${ }^{24}$ ressalta que os espaços de educação popular em saúde constituem um campo de reflexões e práticas que questiona as iniciativas educativas verticalizadas e normatizadoras na prática da promoção da saúde, contemplando a participação popular como estratégia para a conquista da integralidade na atenção e o estímulo à criticidade e à ação sobre a realidade social, o que é também firmado por Paulo Freire $^{14}$ quando diz que "Não é no silêncio que os homens se fazem, mas na palavra, no trabalho, na ação-reflexão".

Nesta perspectiva e com base nas entrevistas realizadas percebeu-se que o trabalho desenvolvido nas rodas de conversa gera benefícios na promoção do autocuidado, transformando o cotidiano dos sujeitos que delas participam. O método utilizado nesse espaço de construção coletiva eleva relações harmoniosas com a equipe de saúde, fortalecendo vínculos terapêuticos e o protagonismo das usuárias envolvidas.

Foi possível perceber que as entrevistadas estão receptivas a essa abordagem de educação em saúde, sendo necessário o fortalecimento das tecnologias leves do cuidado juntamente com demais metodologias ativas que instiguem e proporcionem alternativas de cuidado, conhecimento e protagonismo junto à população idosa e demais faixas etárias bem como, que esta seja incorporada no processo de trabalho como práxis sistemática de atenção à saúde da população na APS.

Observou-se no decorrer da pesquisa que a utilização de entrevistas com questionário semiestruturado para obtenção dos dados, mostrou-se um tanto limitada. Sugere-se para próximas pesquisas da mesma natureza a associação de entrevistas com observação participante e/ ou grupo focal, que permitam um maior espaço de reflexão das participantes sobre a temática, expressões e depoimentos mais amplos e integrais.

\section{REFERÊNCIAS}

1. Tasca R. (Coord.). A atenção à saúde coordenada pela APS: construindo as redes de atenção no SUS - Contribuições para o debate. Organização Pan-Americana da Saúde. [Internet]. Brasília: Organização Pan-Americana da Saúde, 2011. 113 p. (NAVEGADORSUS 2). [Acesso em 20 nov. 2016]. Disponível em: http://www.paho.org/bra/index. php?option $=$ com_docman \&view $=$ download\&alias $=1366$ a-atencao-a-saude-coordenada-pela-aps-construindo-asredes-atencao-no-sus-serie-navegadorsus-n-2-6\&category slug $=$ serie-navegadorsus $-239 \&$ Itemid $=965$

2. Mendes EV. O cuidado das condições crônicas na atenção primária à saúde: o imperativo da consolidação da estratégia da saúde da família. [Internet]. Brasília: Organização PanAmericana da Saúde, 2012. 512p. [Acesso em 20 nov. 2016]. Disponível em: http://bvsms.saude.gov.br/bvs/publicacoes/ cuidado_condicoes_atencao_primaria_saude.pdf
3. Ministério da Saúde. Brasil. Secretaria de Atenção à Saúde. Departamento de Atenção Básica. Envelhecimento e saúde da pessoa idosa/Ministério da Saúde, Secretaria de Atenção à Saúde. [Internet]. Brasília, 2006. [Acessoo em 20 nov. 2016]. Disponível em: http://bvsms.saude.gov.br/bvs/ publicacoes/envelhecimento_saude_pessoa_idosa_n19.pdf

4. Ministério da Saúde. Brasil Secretaria de Atenção à Saúde. Departamento de Atenção Básica. Estratégias para o cuidado da pessoa com doença crônica: obesidade. [Internet]. Brasília: Ministério da Saúde, 2014. 212 p.: il. [Acesso em 20 nov. 2016]. Disponível em: http://bvsms. saude.gov.br/bvs/publicacoes/estrategias_cuidado_doenca_ cronica_obesidade_cab38.pdf

5. Merhy EE. Em busca do tempo perdido: a micropolítica do trabalho vivo. In: Merhy EE, Onocko R. (Org.). Agir em saúde: um desafio para o público. São Paulo: Hucitec; 2007. p. 71-112.

6. Ministério da Saúde. Brasil. Política Nacional de Atenção Básica. Secretaria de Atenção à Saúde. Departamento de Atenção Básica. [Internet]. Brasília, 2012. [Acesso em 26 nov. 2016]. Disponível em: http://189.28.128.100/dab/docs/ publicacoes/geral/pnab.pdf

7. Dias VP, Silveira DT, Witt RR. Educação em saúde: o trabalho de grupos em atenção primária. Rev. APS, 2009. abr./jun.;12(2):221-27.

8. Campos GWS. Saúde Paidéia. São Paulo: Hucitec, 2010.

9. Brasil. Presidência da República. Casa Civil. Subchefia para assuntos jurídicos. Lei no 11.129 de 30 de junho de 2005. Institui a Residência em Área Profissional de Saúde e cria a Comissão Nacional de Residência Multiprofissional em Saúde - CNRMS. 2005. [Internet]. [Acesso em 10 dez. 2016]. Disponível em: http://www.planalto.gov.br/ ccivil 03/ Ato2004-2006/2005/Lei/L11129.htm

10. Tajra I. Roda de conversa como instrumento para criação de grupos de interação social e educacional em saúde relato de experiência [monografia]. Teresina (PI): Universidade Federal do Rio Grande do Sul - UFRGS, Escola de Enfermagem; 2015.

11. Minayo MCS (Org.). Pesquisa social: teoria, método e criatividade. 29a ed. Petrópolis, RJ: Vozes; 2010.

12. Rozendo AS, Justo JS, Correa MR. Protagonismo político e social na velhice: cenários, potências e problemáticas. Revista Kairós Gerontologia (São Paulo). 2010 jun.;13(1): 35-52.

13. Campos GWS, Figueiredo MD, Pereira Júnior N, et al. Application of Paideia methodology to institutional support, matrix support and expanded clinical practice. Interface (Botucatu). 2014;18(Supl 1):983-95.

14. Freire P. Pedagogia da autonomia: saberes necessários à prática educativa. 25 $5^{\mathrm{a}}$ ed. Coleção Leitura. São Paulo: Paz e Terra, 2010.

15. Ministério da Saúde. Brasil. Secretaria de Atenção à Saúde. Departamento de Atenção Básica. Saúde Mental. Departamento de Ações Programáticas Estratégicas. [Internet]. Brasília/DF: Ministério da Saúde, 2013. [Acesso em 10 dez. 2016]. Disponível em: http://bvsms.saude.gov. br/bvs/publicacoes/cadernos_atencao_basica_34 saude mental.pdf

16. Lopes RF, Lopes MTF, Camara VD. Entendendo a solidão do idoso. RBCEH (Passo Fundo). 2009 set./dez.;6(3):373-81.

17. Fekete GM. O envelhecimento da população mundial resumo. Web artigos. [Internet]. 2010. [Acesso em 20 nov. 2016]. Disponível em: http://www.webartigos. com/artigos/o-envelhecimento-da-populacao-mundial/ 37908/\#ixzz4QpgSOwWD 
18. Baquero RVA. A situação das Américas: democracia, capital social e Empoderamento. Empoderamento: instrumento de emancipação social? - uma discussão conceitual. Revista Debates (Porto Alegre). 2012 jan.-abr.; 6(1):173-87.

19. Meneguessi GM, Teixeira JPDS, Jesus CAC de, et al. Reabilitação na lesão medular: reflexão sobre aplicabilidade da teoria do déficit do autocuidado de orem. [Internet]. Rev. enferm. UFPE 2012 dez.;6(12):3006. [Acesso em 28 nov. 2016]. Disponível em: http://www.revista.ufpe.br/ revistaenfermagem/index.php/revista/article/viewFile/2801/ pdf_1779

20. Sampaio J, Santos GC, Agostini M, et al. Limites e potencialidades das rodas de conversa no cuidado em saúde: uma experiência com jovens no sertão pernambucano. Interface (Botucatu). 2014;18(suppl. 2):1299. 311.
21. Barata RB. Acesso e uso de serviços de saúde considerações sobre os resultados da Pesquisa de condições de vida 2006. São Paulo em Perspectiva. 2008 jul./dez.;22(2):19-29.

22. Bonetti OP, Pedrosa JIS, Siqueira TCA. Educação Popular em Saúde como política do Sistema Único de Saúde. Rev. APS, 2011;14(4):397-407.

23. Ministério da Saúde. Brasil. Gabinete do Ministro. Portaria no 2.761, de 19 de novembro de 2013. Institui a Política Nacional de Educação Popular em Saúde no âmbito do Sistema Único de Saúde (PNEPS-SUS). [Internet]. Brasília, 2013a. [Acesso em 12 nov. 2016]. Disponível em: http://bvsms.saude.gov.br/bvs/saudelegis/gm/2013/ prt2761_19_11_2013.html

24. Patrocínio WP, Torres SVS, Guariento ME. Programa de educação popular em saúde: hábitos de vida e sintomas depressivos em idosos. Rev. Bras. Geriatr. Gerontol. (Rio de Janeiro). 2013;16(4):781-92. 